\title{
Risk factors and preventive measures of cerebral hyperperfusion syndrome after carotid artery interventional therapy
}

\author{
SHIBO WANG ${ }^{1 *}$, JING HAN $^{2 *}$, LEI CHENG $^{1}$ and NENGPENG $\mathrm{LI}^{3}$ \\ Departments of ${ }^{1}$ Neurosurgery and ${ }^{2}$ Magnetic Resonance Imaging, Tianjin Huanhu Hospital, Tianjin 300350; \\ ${ }^{3}$ Department of Neurosurgery, Chengdu Aerospace Hospital, Chengdu, Sichuan 610100, P.R. China
}

Received March 20, 2017; Accepted July 5, 2017

DOI: $10.3892 /$ etm.2017.4796

\begin{abstract}
This study sought to investigate the risk factors for cerebral hyperperfusion syndrome (CHS) after carotid artery interventional therapy, and to explore potential preventive measures. Three hundred and eighty-two patients treated with carotid artery stenting at the Huanhu Hospital (Tianjin, China) between January 2010 and January 2016 were divided into CHS and non-CHS groups. A retrospective analysis of patient clinical data was made. The CHS group had more patients presenting coronary heart disease, diabetes, progressive neurological disease and transient recurrent cerebral hemorrhage than the non-CHS group. More patients in the CHS group presented stenosis of the internal carotid artery siphon. More CHS group patients showed plaque formation extending $>3 \mathrm{~cm}$ to the distal end of the internal carotid artery. Finally, more CHS group patients had pressure gradients $>60 \mathrm{mmHg}(\mathrm{p}<0.05)$. Logistics regression analysis showed that preoperative diabetes mellitus and carotid pressure gradient $\geq 60 \mathrm{mmHg}$ were independent risk factors for CHS ( $\mathrm{p}<0.05)$. The ROC curve of carotid pressure gradients $\geq 60 \mathrm{mmHg}$ were made to predict $\mathrm{CHS}$, with the area under curve being $0.949(\mathrm{p}<0.05)$. The best cut-off value was $60 \mathrm{mmHg}$. Therefore, preoperative diabetes and a carotid pressure gradient $\geq 60 \mathrm{mmHg}$ are risk factors for $\mathrm{CHS}$, and these indicators need to be examined prior to operation.
\end{abstract}

\section{Introduction}

Ischemic stroke is a common cerebrovascular disease and has become a major threat to the health and life of the elderly in the modern society. The incidence rate in China is on the rise and the harm to patients has also become more and more

Correspondence to: Dr Shibo Wang, Department of Neurosurgery, Tianjin Huanhu Hospital, 6 Jizhao Road, Jinnan, Tianjin 300350, P.R. China

E-mail: btr75b@163.com

*Contributed equally

Key words: carotid angioplasty and stenting, hyperfusion syndrome, carotid stenosis, risk factors serious (1). The pathological changes caused by carotid artery stenosis are closely related to those observed following ischemic stroke (2). Therefore, using carotid artery stenting (CAS) treatment for extracranial carotid artery stenosis and the prevention of ischemic stroke is important for reducing the threat of cerebrovascular diseases $(3,4)$. However, this operation may lead to CHS. Although the complication rate is low, CHS can be life-threatening, and thus requires close attention from physicians. This study focused on postoperative CHS risk factors for CAS and analyzed a number of preventive measures.

\section{Patients and methods}

Subjects. In total, 382 cases treated using CAS at the Huanhu Hospital between January 2010 and January 2016 were included for retrospective analysis. Patient inclusion criteria are defined as follows: Diagnosed with a transient ischemic attack or cerebral infarction prior to CAS surgery; confirmed to have extracranial carotid artery stenosis prior to CAS surgery; independent acceptance of CAS surgery, with a self-expandable stent placed in the carotid artery sinus; a first postoperative review showing residual stenosis $<30 \%$; complete medical records. Exclusion criteria were as follows: Simultaneous conductance of posterior circulation vascular stenting or a stent placed in the non-carotid sinus; important organ dysfunction; presence of tumor, tendency of bleeding and antiplatelet drug allergy. Based on cerebral hyperperfusion syndrome (CHS) occurrence after surgery, patients were divided into CHS (17 cases) and non-CHS groups (365 cases). This study was approved by the Ethics Committee of the Huanhu Hospital. Patients signed a written informed consent form. Of the 382 patients, 251 were male and 131 were female, aged 60 to 75 years (average, 68.1 7.5 years).

Diagnostic criteria. The CHS diagnosis was made with reference to the definition given by Appleberg et al (5): After CAS, patients presented ipsilateral temporal, forehead, and orbital pain, with or without nausea, vomiting; detection of ipsilateral focal epilepsy or neurological dysfunction; imaging showing high-perfusion status in recanalized vessels and an increase in cerebral artery blood flow; head computed tomography (CT) or magnetic resonance imaging (MRI) showing no ischemic infarction. 
Table I. Clinical data univariate analysis results $[$ mean \pm SD, n (\%)].

\begin{tabular}{lcccr}
\hline Item & CHS group & Non-CHS group & $\mathrm{t} / \chi^{2}$ & P-value \\
\hline Total number of patients & 17 & 365 & & \\
Age (years) & $68.3 \pm 7.3$ & $68.1 \pm 8.5$ & 0.095 & 0.931 \\
Male/female & $12 / 5$ & $239 / 126$ & 0.188 & 0.664 \\
History of drinking & $10(58.8)$ & $195(53.4)$ & 0.190 & 0.663 \\
History of smoking & $12(70.6)$ & $227(62.2)$ & 0.489 & 0.484 \\
Diabetes & $11(64.7)$ & $133(36.4)$ & 5.526 & 0.019 \\
High blood pressure & $12(70.6)$ & $223(61.1)$ & 0.618 & 0.432 \\
Hyperlipidemia & $6(35.3)$ & $118(32.3)$ & 0.065 & 0.799 \\
History of heart disease & $3(17.6)$ & $42(11.5)$ & 0.589 & 0.443 \\
Coronary disease & $12(70.6)$ & $151(41.4)$ & 5.669 & 0.017 \\
Progressive neurological disease & $8(47.1)$ & $83(22.7)$ & 5.294 & 0.021 \\
Recurrent transient cerebral hemorrhage & $5(29.4)$ & $21(5.7)$ & 14.333 & $<0.001$ \\
\hline
\end{tabular}

Study methods. A retrospective survey was conducted to summarize potential risk factors for postoperative CHS. Statistical indicators included patient sex, age, preoperative diseases and perioperative detection indicators.

Statistical analysis. The data were processed by SPSS 19.0 statistical software (IBM, Armonk, NY, USA). Quantitative data are shown mean $\pm \mathrm{SD}$. Qualitative data are presented as ratios, tested using t-tests and Chi-square tests. Independent factor analysis was conducted by logistic regression. The predictive effect of the preoperative carotid artery pressure gradient on postoperative CHS was measured with an ROC curve. $\mathrm{P}<0.05$ was considered to indicate a statistically significant difference.

\section{Results}

Treatment results. No significant adverse reactions were observed in the non-CHS group after CAS. There were 2 cases of death in the CHS group, with the cause of death being uncontrollable cerebral hemorrhage in both cases. One case of subarachnoid hemorrhage was well controlled, with the patient being discharged after recovery. Three cases of focal neurological dysfunction and 11 cases of headache or severe headache were treated correspondingly, with each patient being discharged after recovery.

\section{Univariate analysis}

Comparison of preoperative general information. The total sample size was set as the total number of patients. A comparison showed that there was no significant difference between the two groups in terms of sex, age and history of smoking and drinking $(\mathrm{p}>0.05)$. The CHS group had higher incidence of coronary heart disease, diabetes, progressive neurological diseases and recurrent episodes of transient cerebral hemorrhage prior to surgery. The difference was statistically significant $(\mathrm{p}<0.05$; Table I).

Comparison of perioperative indicators. The total number of samples was set to the total number of CAS cases. The CHS
Table II. Comparison of perioperative indicators [mean $\pm \mathrm{SD}$, n $(\%)]$.

\begin{tabular}{|c|c|c|c|c|}
\hline Items & $\begin{array}{l}\text { CHS } \\
\text { group }\end{array}$ & $\begin{array}{l}\text { Non-CHS } \\
\text { group }\end{array}$ & $t / \chi^{2}$ & P-value \\
\hline No. of CAS cases & 17 & 365 & & \\
\hline $\begin{array}{l}\text { Stenosis grade on } \\
\text { the operated side }\end{array}$ & $78.5 \pm 7.5$ & $79.3 \pm 8.2$ & -0.395 & 0.763 \\
\hline $\begin{array}{l}\text { Stenosis at the carotid } \\
\text { artery siphon }\end{array}$ & $12(70.6)$ & $141(36.9)$ & 7.808 & 0.005 \\
\hline $\begin{array}{l}\text { Plaque extension } \geq 3 \mathrm{~cm} \\
\text { towards the distal end of } \\
\text { the internal carotid artery }\end{array}$ & $10(58.8)$ & $105(27.5)$ & 7.791 & 0.005 \\
\hline $\begin{array}{l}\text { Preoperative carotid } \\
\text { artery pressure gradient } \\
>60 \mathrm{mmHg}\end{array}$ & $17(100.0)$ & $97(25.4)$ & 44.391 & $<0.001$ \\
\hline
\end{tabular}

group consisted of 17 cases of surgery and the non-CHS group consisted of 365 cases of surgery. A comparison showed that there was no significant differences in stenosis grade between the two groups ( $\mathrm{p}>0.05)$. In the CHS group, the internal carotid artery siphon also presented stenosis, the plaque extended within $\geq 3 \mathrm{~cm}$ of the distal end of the internal carotid artery, and there was a higher incidence of carotid artery pressure gradient being $>60 \mathrm{mmHg}$. The differences were statistically significant $(\mathrm{p}<0.05$; Table II).

Logistics regression analysis of CHS risk factors. The number of patients was set to be the sample size. The occurrence of CHS was the dependent variable (yes $=1$, no $=0$ ). Items that were found to be statistically significant during univariate analysis were independent variables (preoperative diabetes: yes $=1$, no $=0$; preoperative coronary heart disease: yes $=1$, no $=0$; preoperative neurological disease: yes $=1$, 
Table III. Logistics regression analysis of CHS risk factors.

\begin{tabular}{lccccc}
\hline Factors & $\beta$ & Wald $^{2}$ & P-value & OR & $95 \%$ CI \\
\hline $\begin{array}{l}\text { Preoperative } \\
\text { diabetes }\end{array}$ & 1.771 & 3.771 & 0.013 & 5.877 & $2.335-17.715$ \\
$\begin{array}{l}\text { Carotid artery } \\
\text { pressure gradient } \\
>60 \mathrm{mmHg}\end{array}$ & 3.058 & 8.258 & 0.000 & 21.285 & $7.171-121.317$ \\
\hline
\end{tabular}

CHS, cerebral hyperperfusion syndrome; CI, confidence interval.

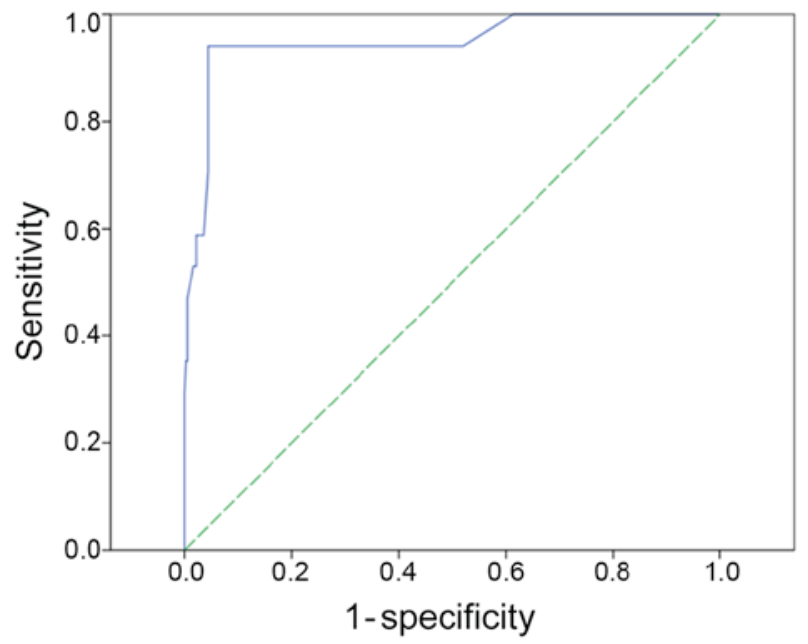

Figure 1. Predictive effect analysis of preoperative carotid artery pressure gradient on CHS. CHS, cerebral hyperperfusion syndrome.

no $=0$; preoperative transient recurrent cerebral hemorrhage: yes $=1$, no $=0$; operative diagnosis of plaque extension to within $\geq 3 \mathrm{~cm}$ of the distal end of internal carotid artery: yes $=1$, no $=0$; preoperative diagnosis of carotid artery pressure gradient $>60 \mathrm{mmHg}$ : yes $=1$, no $=0$ ). The logistics regression analysis showed that preoperative diabetes and carotid artery pressure gradient $>60 \mathrm{mmHg}$ were independent risk factors of cerebral hyperfusion syndrome ( $\mathrm{p}<0.05$; Table III).

Predictive effect analysis of preoperative carotid artery pressure gradient on CHS. The number of cases was the total number of samples. The predicted results for preoperative carotid artery pressure gradient on CHS are shown in Fig. 1. The results showed that the index had a good predictive value for CHS, the area under the curve was 0.949 (standard error $=0.032, \mathrm{p}<0.001,95 \% \mathrm{CI}=0.886-1.000$ ); the best cut-off value was $60 \mathrm{mmHg}$, with a sensitivity of 0.941 and specificity of 0.956 .

\section{Discussion}

Hyperfusion syndrome is mainly seen after carotid artery intervention and the occurrence of this condition is rare but fatal, and is considered a dangerous complication $(5,6)$. A previous study (7) reported 92 cases of CAS patients with 6 cases of complications, and suggested that general anesthesia helped to reduce incidence, while another study (8) reported 180 cases of carotid endarterectomy, where 15 cases had complications, and pointed out that a carotid artery bypass tube could help reduce the incidence rate. This study included 382 CAS patients, 17 of whom presented CHS, demonstrating an occurrence rate consistent with previous studies. Considering that carotid artery intervention is the main treatment of carotid artery stenosis (9), the conclusions above suggest the need for reliable measures to control CHS.

A number of studies have explored the risk factors for this complication, but the conclusions reached were not entirely consistent. A report by Louridas and Jounaid (10) suggested that postoperative hypertension and preoperative diabetes were high risk factors for CHS. This study confirmed that preoperative diabetic patients were more prone to CHS, which may cause hyperglycemia-induced oxidative stress and intracranial vascular endothelial cell injury, leading to endothelial cell dysfunction, endothelial defense damage and endothelial cell gap expansion. As a result, there may be excessive blood filling and increased vascular permeability, potentially triggering CHS $(11,12)$. This suggests that patients with diabetes should pay special attention to the prevention of CHS. This study showed that preoperative hypertension has no definitive value in predicting postoperative CHS, probably because both the CHS and non-CHS groups had high detection rates of hypertension. However, this study, along with others $(13,14)$, still suggests that the postoperative control of blood pressure is very important for the prevention of CHS. This is because after a successful opening of blood vessels with stenosis, a high-blood pressure will rapidly increase the cerebral perfusion pressure, leading to further damage to the cerebrovascular barrier and causing CHS. If the postoperative blood pressure is controlled within the range of 100-140 mmHg, CHS symptoms will gradually disappear with the recovery of cerebrovascular autoregulation $(15,16)$.

Carotid stenosis in patients can also have a certain effect on postoperative CHS. Maramattom (17) pointed out that an incomplete Willis ring, lateral or contralateral carotid artery stenosis, and the application of antiplatelet drugs are also risk factors. The results in this study do not support the above conclusions, although the small CHS sample size here may be a factor. However, this study also pointed out that in the CHS group, internal carotid artery siphon presented stenosis, the plaque extended to within $\geq 3 \mathrm{~cm}$ of the distal end of the internal carotid artery, and there was a higher incidence of carotid artery pressure gradient $>60 \mathrm{mmHg}$. These findings were consistent with the conclusions of Chadha et al (18). These situations can severely affect the preoperative cerebral blood flow reserve, which may be the main cause of CHS.

Our preliminary study (19) showed that the pressure difference at both ends of the carotid artery with stenosis directly affected local blood flow. The greater the pressure difference, the more prominent the effect of improved blood supply after the stenosis is treated. This study showed that for patients with a pressure gradient difference $<60 \mathrm{mmHg}$, the benefits and risks of carotid artery stent angioplasty should be comprehensively evaluated and medical treatment can be considered. However, this study also showed that patients with high pressure differences are at higher risk for postoperative CHS, which was confirmed by ROC curve. When $60 \mathrm{mmHg}$ was used as the threshold to predict postoperative CHS, the 
sensitivity was 0.941 and the specificity was 0.956 . This suggests that the early detection of carotid artery pressure difference may help prevent CHS. For patients whose pressure gradient is too low or too high, we would need to consider other treatment options. The use of a pressure guide wire can effectively monitor a patient's carotid artery pressure gradient (20). Although a ROC curve can be used to obtain the boundary value of the carotid artery pressure gradient, as was done in this study, the reliability of the conclusions may need further confirmation due to the small sample size.

In conclusion, preoperative diabetes and a carotid artery pressure gradient $>60 \mathrm{mmHg}$ are risk factors for hyperfusion syndrome after carotid artery intervention. Strict analysis of preoperative factors can help prevent hyperfusion syndrome.

\section{References}

1. Wang Y,Liao X, Zhao X, Wang DZ, Wang C, Nguyen-Huynh MN, Zhou Y, Liu L, Wang X, Liu G, et al; China National Stroke Registry Investigators: Using recombinant tissue plasminogen activator to treat acute ischemic stroke in China: Analysis of the results from the Chinese National Stroke Registry (CNSR). Stroke 42: 1658-1664, 2011.

2. Barnett HJ, Gunton RW, Eliasziw M, Fleming L, Sharpe B, Gates $\mathrm{P}$ and Meldrum $\mathrm{H}$ : Causes and severity of ischemic stroke in patients with internal carotid artery stenosis. JAMA 283: 1429-1436, 2000.

3. Gonzalez I, Kenny D, Slyder S and Hijazi ZM: Medium and long-term outcomes after bilateral pulmonary artery stenting in children and adults with congenital heart disease. Pediatr Cardiol 34: 179-184, 2013.

4. Farooq MU, Goshgarian C, Min J and Gorelick PB: Pathophysiology and management of reperfusion injury and hyperperfusion syndrome after carotid endarterectomy and carotid artery stenting. Exp Transl Stroke Med 8: 7, 2016.

5. Appleberg M, Cottier D, Crozier J, Graham J and Lane R: Carotid endarterectomy for asymptomatic carotid artery stenosis: Patients with severe bilateral disease a high risk subgroup. Aust N Z J Surg 65: 160-165, 1995.

6. Isozaki M, Arai Y, Higashino Y, Okazawa H and Kikuta KI: Cerebral hyperperfusion syndrome resulting in subarachnoid hemorrhage after carotid artery stenting. Ann Nucl Med 30: 669-674, 2016
7. Van Stavern RB and Chaturvedi S: Evolving treatment strategies for carotid artery stenosis. Curr Treat Options Cardiovasc Med 6: 105-112, 2004

8. Berland TL, Cayne NS and Veith FJ: Symptomatic carotid artery stenosis is best treated by carotid endarterectomy. Methodist DeBakey Cardiovase J 7: 41-43, 2011.

9. Bhattacharya P and Chaturvedi S: Intensive medical therapy for asymptomatic carotid artery stenosis. Curr Cardiol Rep 13: $18-23,2011$

10. Louridas G and Junaid A: Management of carotid artery stenosis. Update for family physicians. Can Fam Physician 51: 984-989, 2005.

11. Young KC, Holloway RG, Burgin WS and Benesch CG: A cost-effectiveness analysis of carotid artery stenting compared with endarterectomy. J Stroke Cerebrovasc Dis 19: 404-409, 2010.

12. Abbas SM, Adams D and Vanniasingham P: What happens to the external carotid artery following carotid endarterectomy? BMC Surg 8: 20, 2008

13. Sato K, Yamada M, Kuroda H, Yamamoto D, Asano Y, Inoue Y, Fujii $\mathrm{K}$ and Kumabe T: Time-of-flight MR angiography for detection of cerebral hyperperfusion syndrome after superficial temporal artery-middle cerebral artery anastomosis in Moyamoya disease. AJNR Am J Neuroradiol 37: 1244-1248, 2016.

14. Pinho J, Amorim JM, Alves JN, Araújo JM and Ferreira C: Cerebral hyperperfusion syndrome after internal carotid artery dissection with persistent occlusion. Neurol Sci 37: 1175-1177, 2016.

15. Yoshie T, Ueda T, Takada T, Nogoshi S, Fukano T and Hasegawa Y: Prediction of cerebral hyperperfusion syndrome after carotid artery stenting by CT perfusion imaging with acetazolamide challenge. Neuroradiology 58: 253-259, 2016.

16. Uchida K, Yoshimura S, Shirakawa M, Shindo S, Egashira Y, Iwama $\mathrm{T}$ and Yamada K: Experience of staged angioplasty to avoid hyperperfusion syndrome for carotid artery stenosis. Neurol Med Chir (Tokyo) 55: 824-829, 2015.

17. Maramattom BV: Cerebral hyperperfusion syndrome after intracranial stenting of the middle cerebral artery. Indian J Crit Care Med 20: 620-621, 2016.

18. Chadha DS, Singh N, Tewari AK, Kumar RS, Yadav KK, Naveen AJ, Bhartiya M, Gupta VK, Wagh A and Ghosh AK: Hyperperfusion syndrome after carotid artery stenting. Med J Armed Forces India 71 (Suppl 1): S156-S159, 2015.

19. Feng T, Han J, Jia Q, Li XD, Wang SB and Fan YM: Application of pressure wire in carotid artery stenting. Chin J Contemp Neurol Neurosurg 13: 3, 2013 (In Chinese).

20. Hannawi B, Lam WW and Younis GA: Pressure wire used to measure gradient in chronic mesenteric ischemia. Tex Heart Inst J 39: 739-743, 2012. 